\title{
KILLING RATIONAL CHARACTERISTIC CLASSES BY SURGERY
}

\author{
by STAVROS PAPASTAVRIDIS
}

(Received 8 July 1979)

\begin{abstract}
0. Introduction. Let $f_{r}: X_{r} \rightarrow B O(r)$ be a sequence of fibrations with maps $g_{r}: X_{r} \rightarrow$ $X_{r+1}$ such that the usual diagram commutes. For such a situation R. Lashof defines the concept of an $X$-structure on manifolds (see [3]), and proves a Thom-isomorphism for the cobordism groups of such manifolds. Let $n, m$ be positive integers which are fixed throughout this paper. If $r$ is very big in comparison with $n+m$ then $X_{r}$ is a simply connected CW-complex and the map $\left(g_{r}\right)_{*}: H_{*}\left(X_{r} ; Q\right) \rightarrow H_{*}\left(X_{r+1} ; Q\right)$ is an isomorphism up to dimension $n$. Let $\gamma$ be the pull-back over $X_{r}$ of the universal $r$-linear bundle (which is, of course, a bundle over $B O(r))$. If $r$ is very big in comparison with $n+m$, then we put $X=X_{r}$, and we assume that $\gamma$ is orientable and oriented. The elements of $H^{*}(X ; Q)$ of dimension not greater than $n$, will be called rational universal $X$-characteristic classes. It is well-known that many of the usual classes of manifolds may be described in terms of $X$-structures, (e.g. SO, SU, Spin-manifolds etc.).
\end{abstract}

Let $M$ be a closed, compact, differentiable manifold with an $X$-structure. Such a manifold will be called an $X$-manifold. If $r$ is very big in comparison with the dimension of $M$ then the $X$-structure of $M$ defines a map $\nu_{M}: M \rightarrow X$ (i.e. a lifting of the Gauss map $M \rightarrow B O(r))$ which is well-defined up to homotopy. Via the map $\nu_{M}$ we can define $X$-characteristic numbers and $X$-characteristic classes of the $X$-manifold $M$.

Let $S \subseteq H^{*}(X ; Q)$ be a set of $X$-characteristic classes of dimension at most $n$. Let $S$ contain $m$ elements.

Condrion 1. The elements of $S$ are cohomology classes of even dimension. Further we can put the elements of $S$ in an ordering, i.e. $S=\left\{u_{1}, u_{2}, \ldots u_{m}\right\}$, such that the following things happen:

(i) If $a \in H^{*}(X ; Q), \operatorname{dim}\left(a \cdot u_{1}\right) \leqslant n+1$ and $\left(a \cdot u_{1}\right)=0$, then $a=0$.

(ii) If $i=2,3, \ldots, k, a \in H^{*}(X ; Q), \operatorname{dim}\left(a \cdot u_{i}\right) \leqslant n+1$, and $a \cdot u_{i}$ belongs to the ideal generated by $u_{1}, u_{2}, \ldots, u_{i-1}$, then $a$ belongs to the ideal generated by $u_{1}, u_{2}, \ldots, u_{i-1}$.

THEOREM 2. Let $M$ be an $n$-dimensional $X$-manifold such that all its $X$-characteristic numbers which include a class of $S$ as a factor vanish. If the set $S$ satisfies Condition 1, then $M$ is $X$-cobordant to a manifold which has all the classes of $S$ zero.

Let $q$ be a non-negative integer which is not greater than $n$.

Condition 3. Let $H^{*}(X ; Q)$ be a polynomial algebra up to dimension $n+1$, with even dimensional generators. Let $A$ be the ideal of $H^{*}(X ; Q)$ generated by all the elements of dimension not greater than $q$. Let $p^{*}: H^{*}(X ; Q) \rightarrow H^{*}(X ; Q) / A$ be the obvious projection. We assume that the set $p^{*}(S) \subseteq H^{*}(X ; Q) / A$ satisfies Condition 1 , (i.e. We can put its elements in an ordering, $p^{*}(S)=p^{*}\left(u_{1}\right), p^{*}\left(u_{2}\right), \ldots, p^{*}\left(u_{m}\right)$, etc.).

THEOREM 4. Let $M$ be an $n$-dimensional $X$-manifold, such that all its $X$-characteristic numbers which include a class of $S$ as a factor vanish, and its stable normal bundle is

Glasgow Math. J. 21 (1980) 209-212. 
$q$-trivial (i.e. The restriction $\nu_{M} \mid M^{q}$ to the q-skeleton is homotopically trivial). Then $M$ is $X$-cobordant to a manifold which has all the classes of $S$ zero, and its stable normal bundle is q-trivial.

Theorems 2,4 were proved by R. Lashof in the special case that $X=B U(r / 2)$ and $S$ contains one element i.e. $m=1$. This paper grew out of an attempt to understand better Lashof's results. As an easy consequence of Theorems 2,4, we have:

Corollary 5. Let $H^{*}(X ; Q)$ be a polynomial algebra of even dimensional generators. Let $u \in H^{*}(X ; Q)$ be an $X$-characteristic class and let $M$ be an $X$-manifold such that all its characteristic numbers including $u$ as a factor are zero. Then $M$ is $X$-cobordant to a manifold $M^{\prime}$ which has the class u equal to zero. Further if the stable normal bundle of $M$ is $q$-trivial, the same may be assumed for $\mathbf{M}^{\prime}$.

I don't know whether the conditions imposed on $S$ are really necessary, at least for the case where $H^{*}(X ; Q)$ is a polynomial algebra of even dimensional generators. Perhaps this is an interesting question to investigate.

1. How to kill one class. In this section we will prove Theorems 2,4 for the case where $S$ contains just one element i.e. $S=\{u\}$. But first we need some preparation.

LEMMA 1.1. If all the rational $X$-characteristic numbers of an $X$-manifold are zero, then some finite multiple of this manifold is an X-boundary.

Proof. It follows from the fact that in the stable range the rational Hurewicz homomorphism is an isomorphism, so we have the isomorphism

$$
\pi_{r+n}(T(\gamma)) \otimes Q \rightarrow H_{r+n}(T(\gamma) ; Q) .
$$

Let $u_{z} \in H^{*}(X ; Z)$ be an integral class whose rational reduction is the class $u$ (of course this class may not be uniquely defined). Let $X(u)$ be the space that we get from $X$ by killing a finite multiple of $u_{z}$ (any one) by the usual method of paths fibration. Of course the space $X(u)$ is not uniquely defined, because it depends upon the choice of $u_{z}$ and its finite multiple, but whatever we say about this space is true for all the choices.

Lemma 1.2. Let $f: X(u) \rightarrow X$ be the well-known fibration, and let us assume that Condition 1 is satisfied (i.e. for the case $m=1, S=\{u\}$ ). Then the kernel of the map $f^{*}: H^{*}(X ; Q) \rightarrow H^{*}(X(u) ; Q)$ is the ideal generated by $u$. Further the map $f^{*}$ is onto up to dimension $n$.

Proof. It follows by applying standard (Serre)-spectral sequence arguments on the fibration $f$.

Note that Condition 1(i) is exactly what is needed to guarantee that the map $f^{*}$ is onto up to dimension $n$.

Let $p: X_{q} \rightarrow X$ be the $q-$ connective covering of $X$ (for details see [2], p. 155).

Lemma 1.3. Let us assume that Condition 3 is satisfied. Then the kernel of the map $p^{*}: H^{*}(X ; Q) \rightarrow H^{*}\left(X_{q} ; Q\right)$ is the ideal generated by the elements of $H^{*}(X ; Q)$ of degree at most $q$. Further, the map $p^{*}$ is onto up to dimension $n$. 
The last Lemma will follow immediately from the following:

Lemma 1.4. Let $g: X_{i} \rightarrow X_{i-1}$ be the obvious fibration, $\left(i=1,2, \ldots, q\right.$ and $\left.X_{0}=X\right)$. Let us assume that Condition 3 is satisfied. Then the kernel of $\mathrm{g}^{*}$ is generated by all the elements of $H^{*}\left(X_{i-1} ; Q\right)$ of dimension at most $i$. Further the map $\mathrm{g}^{*}$ is onto up to dimension $n$.

Proof. From the definition of connective fiberings, it follows easily that the fibre of $g$ is $K\left(i-1, \pi_{i}(X)\right)$. And the result follows by (Serre)-spectral sequence arguments on the fibration $g$.

Note that Condition 3 is essential for the "onto" part of the two previous Lemmas.

LeMma 1.5. The map $\nu_{M}: M \rightarrow X$ admits a lifting $\nu_{M}^{q}: M \rightarrow X_{q}$ if and only if its restriction $\nu_{M} \mid M^{a}$ to the $q$-skeleton of $M$ is homotopically trivial.

Proof. It follows from the definition of connective fiberings (see [2], p. 155), and standard obstruction theory arguments.

Now we are in position to apply R. Lashof's geometrical arguments (see [3], section 4) to finish the proof of Theorems 2,4 for the case that the set $S$ contains one characteristic class. So, from now on till the end of this section, we assume that $S=\{u\}$, Condition 1 is satisfied and that $u$ annihilates all the $X$-characteristic numbers of $M$.

LEMMA 1.6 If $\nu_{m}^{*}(u)$ is a spherical cohomology class of $H^{*}(M ; Q)$, then $u$ can be killed by $X$-cobordism.

Proof. Since $\nu_{M}^{*}(u)$ is spherical, then there is a map $f: M \rightarrow S^{\operatorname{dim} u}$ such that $f^{*}(i)=$ $\nu_{M}^{*}(u)$, where $i \in H^{\operatorname{dim} u}\left(S^{\operatorname{dim} u} ; Q\right)$ is the generator. Deforming $f$ to be differentiable and transversal to the base point $p$ of $S^{\text {dim } u}$, we have $f^{-1}(p)$ to be a submanifold $N$, imbedded in $M$. Clearly $\operatorname{dim} N+\operatorname{dim} u=n$, the normal bundle of $N$ in $M$ is trivial and $N$ represents an homology class of $M$ which is dual to $\nu_{M}^{*}(u)$. Since the normal bundle of the imbedding is trivial, then $N$ admits naturally an $X$-structure, and it can be proved, (see [3], Proposition 4.1) that all the rational $X$-characteristic numbers of $N$ are zero. So, by Lemma 1.1, some finite multiple $t$ of $N$ is an $X$-boundary. Since $N$ is imbedded in $M$ with trivial normal bundle, we may imbed $t$ disjoint copies of $N$ parallel to each other in the product neighborhood of $N$ in $M$, and hence their connected sum $t N$. So, the $X$-manifold $t N$ is connected, it is embedded in $M$ with trivial normal bundle, it is an $X$-boundary and finally it represents the homology class which is dual to $\nu_{M}^{*}(u)$. We put $t N=N_{1}$.

So we have an imbedding $N_{1} \times D^{\text {dimu }} \subseteq M$. It can be proved (see [3], Proposition 4.2) that the natural $X$-structure of $M-\left(N_{1} \times \operatorname{Int} D^{\mathrm{dim} u}\right)$ can be lifted to an $X(u)$-structure. This $X(u)$-structure gives an $X(u)$-structure on $N_{1}$. By Lemma 1.2, all the rational $X(u)$ characteristic numbers of $N_{1}$ are zero, so some finite multiple $s$ of $N_{1}$ is an $X(u)$ boundary. Again we take $s$ copies of $N_{1}$ and we imbed them parallel to each other in the product neighborhood of $N_{1}$ in $M$. So we have the connected sum $s N_{1}$ imbedded in $M$ with trivial normal bundle and representing the homology class which is dual to $\nu_{M}^{*}(u)$. Further $s N_{1}$ is an $X(u)$-boundary. We put $N_{2}=s N_{1}$. 
Now the manifold $N_{2}$ satisfies all the conditions of Proposition 4.4 of [3], so, as R. Lashof proves, we can kill $u$ by $X$-cobordism.

Note that the fact that the obvious map $X(u) \rightarrow X$ is onto in cohomology up to dimension $n$ is essential for the proof.

LEMMA 1.7. Let us assume that $\nu_{M}^{*}(u)$ is a spherical cohomology class of $H^{*}(M ; Q)$, that the stable normal bundle of $M$ is $q$-trivial and that Condition 3 is satisfied. Then $M$ is $X$-cobordant to an $X$-manifold $M^{\prime}$ which has its stable normal bundle q-trivial and such that $\nu_{\mathbf{M}^{\prime}}^{*}(u)=0$.

Proof. The same as the previous Lemma. We use Lemma 1.3.

Now the proof of Theorems $2 ; 4$ for the case $S=\{u\}$ follows easily:

Proof of Theorems 2,4, for the case $S=\{u\}$. We will use a result of I. Berstein [1, Proposition 1.5] which is stated as follows: Let $K$ be a finite dimensional $C W$-complex, $n$ an integer and $h$ an arbitrary element of $H^{n}(K ; Z)$ such that the cup square $h \cup h$ is an element of finite order. Then there exist an integer $N>0$ and a map $f: K \rightarrow S^{n}$, so that $f^{*}(i)=N h$ where $i$ is the fundamental class of $H^{n}\left(S^{n} ; Z\right)$.

Since $M$ is finite dimensional, some $2^{j}$ th power of $\nu_{M}^{*}(u)$ is zero. So by Lemmas 1.6 , 1.7 we can kill $\nu_{M}^{*}(u)^{2 j-1}$. Thus by iterating the preceding argument $j-1$ times we eventually kill $u$.

\section{The general case.}

Proof of Theorem 2,4. Now everything is very easy. We can kill the classes of $S$ successively one-by-one. The Conditions 1,3 guarantee that at each stage the necessary conditions are satisfied so we can kill the next class.

\section{REFERENCES} 9-14.

1. I. Berstein, Homotopy mod C of spaces of category 2, Comment. Math. Helv. 35 (1961),

2. S. T. Hu, Homotopy theory (Academic Press, 1959).

3. R. Lashof, Poincaré duality and cobordism, Trans. Amer. Math. Soc. 109 (1963), 257-277.

4. R. Mosher and M. Tangora, Cohomology operations and applications in homotopy theory (Harper and Row, 1968).

UNIVERSITY OF PATRAS

Patras, Greece 